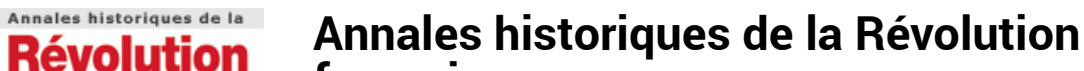

française française

361 | juillet-septembre 2010

Entre scatologie et fantasmes sexuels, le cul et son imaginaire

\section{Le son du corps, ou l'âme en pet}

\section{Philippe Bourdin}

\section{(2) OpenEdition}

1 Journals

\section{Édition électronique}

URL : https://journals.openedition.org/ahrf/11694

DOI : 10.4000/ahrf.11694

ISSN : 1952-403X

Éditeur :

Armand Colin, Société des études robespierristes

\section{Édition imprimée}

Date de publication : 1 septembre 2010

Pagination : 65-90

ISBN : 978-2-200-92633-5

ISSN : 0003-4436

Référence électronique

Philippe Bourdin, "Le son du corps, ou l'âme en pet », Annales historiques de la Révolution française [En ligne], 361 I juillet-septembre 2010, mis en ligne le 01 septembre 2013, consulté le 23 avril 2022. URL : http://journals.openedition.org/ahrf/11694; DOI : https://doi.org/10.4000/ahrf.11694 


\title{
LE SON DU CORPS, OU L'ÂME EN PET
}

Philippe BOURDIN

\begin{abstract}
Il est de ces vents passagers qui portent, incontinents et inconstants, un vague relent d'histoire. Échappés des humaines entrailles, ils rappellent au musicien de l'instant qu'à trop faire l'homme il fait la bête, balayant de son souffle pestilentiel des siècles de civilité; au libertin, sinon au coprophage, que toutes les entrées en matière ne se valent pas. Plus musicaux, ces instruments des pétomanes unissent des sociétés masculines minoritaires que la Révolution n'éradique pas : trop fermés pour ne pas manquer d'air, les clubs de francs péteurs revendiquent tambours et trompettes. Dans le privé partagé d'académies pétulantes, comme dans le roman ou le théâtre qui en rapportent miasmes et constipations, on se fait cependant fort de se distinguer du bas peuple et de savoir dresser une carte sociale des inconvenances. La Révolution, du moins dans les années où se font sentir le plus vivement son souffle libérateur et les talents des caricaturistes, usera de cette dépréciation pour abolir les frontières des ordres et mieux confondre ses ennemis, renvoyés au stade anal ou balayés d'une vesse dédaigneuse.
\end{abstract}

Mots-clés : culture savante, culture populaire, littérature, théâtre, caricature, libertinage, coprophagie.

«Que jamais nus ame n'aport,

Qui de vilain sera issue,

Ne puet estre qu'elle ne pue. »

(Rutebeuf, Dou pet au vilain, XIII ${ }^{\mathrm{e}}$ siècle) 
« Du pet qu'il fist la terre trembla neuf lieues à la ronde, duquel avec l'air corrompu engendra plus de cinquante et troys mille petitz hommes, nains et contrefaitcz, et d'une vesne ${ }^{1}$ qu'il fit engendra autant de petites femmes acropies, comme vous en voyez en plusieurs lieux. »

(Rabelais, Pantagruel, chapitre XXVII, 1532)

Pourquoi moi? Telle est la question qui, la première, me vint in petto à l'esprit lorsque Michel Biard et Pascal Dupuy me proposèrent pour le présent numéro de porter au grand jour ce que la civilité nous a appris depuis des siècles à rendre sournois, sauf à se parer des rougeurs de la gêne ou à rejoindre le concert des buveurs en des sociétés très masculines : le pet. L'expression « libre comme l'air », qui allait de soi pour un objet d'étude aussi volatil et si rétif à toute hauteur de vue, ne m'a jamais semblé aussi contraignante, mesurant notamment combien aucun jeune chercheur ne pouvait en faire un argument de carrière, acceptant au mieux dans le privé d'une impubliable rencontre à prétention scientifique un bizutage à fleuret forcément moucheté. Car les querelles intestines qui déchirent notre milieu sont légion, et j'entends déjà certains se gausser d'une école marxiste revisitant ses fondements, laissant, scrupuleuse à la virgule près, cette trace si particulière dans l'histoire, dans les Annales de surcroît. Mais, d'une part le matérialiste, oubliant les sirènes d'une postérité intellectuelle qui fait parfois bon ménage avec Anastasie, ne peut être tout à fait insensible aux définitifs sons du corps. D'autre part, à l'heure de quitter mes responsabilités à la tête de la Société des études robespierristes, cette ultime pirouette, cul par-dessus cette tête et la vanité de tout pouvoir, ne me déplaît pas. Enfin, l'historien ne saurait ignorer le poids des héritages comportementaux, la culture de la distinction si chère à Norbert Élias, ni manquer de s'interroger sur la diabolisation des flatulences individuelles. Elles traduisent pour bien des générations un Enfer et une corruption intérieurs, cette âme noire et populaire dont parle Rutebeuf. Elles animent aussi les jeux assumés d'une minorité libertine et parfois coprophage, d'un petit peuple que la scatologie fait partir d'un rire que la Révolution entretient politiquement, non sans références littéraires - si Cérutti et ses disciples offrent une lecture policée de Rabelais, ils en font aussi le héraut de 
l'anti-satire contre la prose royaliste ${ }^{2}$. Le Père Duchesne n'a-t-il pas lu les exploits de Pantagruel lorsqu'il fait sortir un «club de pestiférés [...] du cul de la noblesse $»^{3}$ ? Car, pour les révolutionnaires, tout n'est pas Raison, et d'aucuns ne craignent pas de s'asseoir un instant sur les principes, de les offrir à tous vents, si l'on en juge par les pots de chambre qui reproduisent des scènes des journées principales ou des allégories ${ }^{4} \ldots$

\section{Le pet, marqueur social et culturel}

Peut-il cependant exister une quelconque accoutumance aux miasmes individuels dans une société nobiliaire et bourgeoise qui, plus on avance dans le siècle, et jusqu'au Directoire au moins, bannit l'odeur du musc et se méfie des fétidités supposées mortelles, échappées des fossés des villes où stagnent les matières fécales? Adoptant avant l'heure le statut de " témoin oculaire et nasiculaire » revendiqué par le marquis de Villette, grand admirateur de Voltaire, offusqué en 1793 par les dégoûtants cadeaux dont ses contemporains gratifient l'enceinte de son hôtel particulier, Louis-Sébastien Mercier témoigne sans appel sur l'inconvenance des comportements, l'absence de latrines idoines, sinon au Palais-Royal, et les déboires de la privatisation des déjections par les asphyxiantes fosses d'aisance, sûr que « les esprits animaux sont encore dans [nos] excréments $»^{5}$. Faut-il être Sade ou ressortir du peuple ignorant des valeurs aéristes pour se complaire dans « l'évocation archaïsante du bon vieux temps, où les choses avaient leur odeur véritable », dans le respect de la nature ${ }^{6}$ ? Celle, animale, de l'odorat est pourtant fortement dénoncée par Condillac, "parce que c'est de tous les sens celui qui paraît contribuer le moins aux connaissances de l'esprit humain », laissant la statue de l'homme toute à son odeur, se confondant avec

(2) Antoine DE BAECQUe, Les éclats du rire. La culture des rieurs au XVIII siècle, Paris, Calmann-Lévy, 2000, p. 181-184. Pensons, pour le genre sérieux, à la somme que publie Ginguené en 1791 : De l'autorité de Rabelais dans la révolution présente et dans la constitution civile du clergé, ou institutions royales, politiques et ecclésiastiques tirées de Gargantua et de Pantagruel; et, pour l'anti-satire, à la Lettre de Rabelais aux 94 rédacteurs des Actes des Apôtres (avril 1790).

(3) Le Père Duchesne, n 140-1792. Cité par Michel BIARD, Parlez-vous sans-culotte? Dictionnaire du Père Duchesne 1790-1794, Paris, Tallandier, 2009, p. 178.

(4) Lynn Hunt, « Révolution française et vie privée », in Philippe ArIÈs et Georges DuBY (dir.), Histoire de la vie privée, tome 4 : « De la Révolution à la Grande Guerre », Paris, Seuil, 1987, rééd. « Points-Seuil » 1999, p. 25.

(5) Louis-Sébastien Mercier, Le Tableau de Paris, Paris, 1782-1788, rééd. Michel Delon, Paris, « Bouquins »-Robert Laffont, 1990, p. 49 (« L'air vicié »), p. 234-235 (« Latrines publiques »), p. 332-334 (« Latrines »).

(6) Michel Delon, Le savoir-vivre libertin, Paris, Hachette, 2000. 
elle faute d'une intelligence autre ${ }^{7}$. Le mot vesse, «vent qui sort parderrière sans faire de bruit » selon le Dictionnaire de Trévoux, est fort dépréciatif: il stigmatise aussi une femme prostituée, et se décline en vézon dans l'argot lyonnais, où il désigne aussi bien un putois qu'une « pute », vézonner équivalant à lâcher un pet ${ }^{8}$. Pendant que le «marécage des sanies ${ }^{9}$ envahit les rues de Paris, les alentours du château de Versailles, les ruelles étroites et tortueuses de Clermont-Ferrand gravies par Arthur Young, que la science porte loin l'analyse des gaz irrespirables (jusqu'aux prélèvements des émanations intimes des savants), la lutte pour la désodorisation, qui est celle pour l'hygiène publique, pour l'effacement des preuves putrides, suffocantes du corps et de la trahison des humeurs intestines, est alors bien engagée. Nombre des 57 procédés dénombrés entre 1762 et 1853 pour désinfecter les fosses d'aisance reposent sur des recettes pour aromatiser la matière : bergamote, sucs de limons et d'oranges, eau de lavande ou de fleurs d'orangers, clous de girofle, essences et huiles mêlent leurs parfums dans ce but, provoquant le rire des vidangeurs parisiens devant « les pralines à la fleur d'orange » qu'on leur offre - et de se gausser des inventeurs qui finiront par «métamorphoser la merde en alouettes ${ }^{10}$. Dans l'intimité du particulier, le linge est le propre, le parfum nettoie, alors que les bains publics, ouverts dans les années 1780 à Paris, Dijon, Caen et ailleurs, demeurent chers, que la cuvette, utilisée pour des ablutions partielles, ne se répand qu'à la toute fin du siècle dans la population urbaine. Les baignoires, les « chaises de propreté » et les bidets sont, jusque dans la décennie 1780 où certains sont adoptés par la bourgeoisie aisée, réservés à l'élite nobiliaire pour laquelle le coût de l'eau n'est rien; elle a aménagé dès le deuxième tiers du XVIII ${ }^{\mathrm{e}}$ siècle des espaces spécifiques où la toilette demeure ostentatoire, tandis que les traités médicaux des années 1760 invitent à distinguer dans un souci d'hygiène les différentes parties du corps, insistant sur les exhalations particulières de «l'entre fessons ou la raie » (Le Conservateur de la santé, 1763) ${ }^{11}$. D'aucuns assistent pourtant, et s'en flattent, aux efforts royaux pour occuper pleinement le

(7) Condillac, Traité des sensations, « Dessein de cet ouvrage ».

(8) Patrick Wald-Lasowski, La science pratique de l'amour. Manuels révolutionnaires érotiques, Paris, Éditions Philippe Picquier, 1998, p. 260.

(9) Alain Corbin, Le miasme et la jonquille. L'odorat et l'imaginaire social, XVIII XIX $X^{e}$ siècles, Paris, Aubier, 1982, p. 30.

(10) Dominique Laporte, Histoire de la merde, Paris, Christian Bourgeois, 1978, p. 69.

(11) Georges Vigarello, Le propre et le sale. L'hygiène du corps depuis le Moyen-Âge, Paris, Seuil, 1985. 
trône d'un instant, quoique les cabinets à soupape puissent protéger dès Louis XV l'intimité royale (avant que son successeur n'adopte de fort modernes "lieux à l'anglaise »). Sans compter les maladies dudit roi, savamment mises en scène tandis que l'opinion publique agite parfois des hypothèses peu reluisantes sur les conséquences de ses aventures galantes $^{12}$; la rémission d'une dangereuse constipation, survenue en 1744 à Metz, inspire, à Piron sans doute, cette Ode à l'occasion de la convalescence du Roi, L'Étron royal :
«Que vois-je! Ô Ciel! C'est un étron!
Que la matière en est louable!
Il est gros comme un saucisson
Et garnirait bien une table.
C'est l'œuvre du plus grand des rois,
L'odeur, le goût sentent le trône;
Et jamais anus bourgeois
N'en eût accouché sans matrône ».

Foin des pudibonderies ostensibles de la civilité, de la maitrise du corps réglée par les leçons d'Érasme ou les interdits de Jean-Baptiste de La Salle, pour lequel «il est très incivil de laisser sortir des vents de son corps [...], indécent de le faire d'une manière qu'on puisse être entendu des autres ${ }^{13}{ }^{13}$; Combalusier, régent de la faculté de médecine de Paris, en sa Pneumopathologie des maladies venteuses (1754), rend gloire aux stoïciens, qui laissent chacun libre de lâcher des vents par tout temps, par en haut et par en bas ${ }^{14}$. Entretenir de ses humeurs intestines est justement admis d'ordinaire si cela relève de soucis de santé ou des petites confidences entre proches amis, dont le ton peut du reste se faire badin, faute de savoir comment aborder l'irrépressible. Le directeur de la poste aux lettres de Riom, en Auvergne, Gabriel Dubreul, ne cache ainsi rien des flux qui l'assaillent à son ami d'enfance, le futur conventionnel Gilbert Romme, auprès duquel il a l'habitude d'épancher d'ordinaire plus sensiblement son âme. Depuis ses 16 ans, il ne contrôle plus les peurs que les contes et histoires de revenants n'avaient pas réussi jusqu'alors

(12) Bernard Hours, Louis XV, un portrait, Privat, Toulouse, 2009, p. 29-40.

(13) Jean-Baptiste de LA SALLe, Les règles de la bienséance et de la civilité chrétienne, Paris, 1729

(14) Roger-Henri Guerrand, Les lieux. Histoire des commodités, Paris, La Découverte, 1985 , p. 48. 
à instiller en lui. La mort de son père est le déclencheur d'une longue suite d'indispositions adolescentes (évanouissements, saignements de nez, vomissements, tremblements ou tétanisation des muscles, absence de concentration dans l'effort intellectuel). Trois ans durant, elles le laissent « le ventre constipé et n'ayant la tête bien tranquille que lorsque les évacuations se faisoient bien ». Et de préciser, nous renvoyant aux errements des médications : "Lorsqu'à la suite je prends mon lavement, je rends beaucoup de vent et je me sens soulagé. Il est bon de dire que les vents me montent à la tête, que si je n'en fais pas dans la journée, étant au lit, j'y éprouve des mouvements aux doigts des pieds et quelquefois des soubresauts $\gg{ }^{15}$. Or, comme s'il n'y avait pas de fumet sans défunt, ce caput mortuum de nos digestions est justement associé immanquablement à des visions de cadavres, de morts et de mourants... Aux lavements, à l'eau, au son bouilli et au savon, Dubreul ajoute donc un régime alimentaire susceptible, selon lui, d'éviter toute incongruité : des bouillons dégraissés, des viandes rôties, des poissons maigres, du riz, du vin coupé d'eau, mais ni ragoût, ni fricassée, ni épices, ni pâtisserie, ni aucun fruit. Avec cette entorse aux connaissances : «Les jours maigres, je mange des fèves qui ne me fatiguent point malgré qu'elles passent pour être pesantes et venteuses $»^{16}$.

Romme consulte en Russie ou à Paris, inquiet de la chétivité de son comparse, de sa nervosité, conséquences de ses troubles névrotiques. Mais lorsque Dubreul réclame un ouvrage du maître de pension Hurtault, L'Art de péter, essai théorique et méthodique, Gilbert Romme, habitué à de sérieux échanges sur les progrès des sciences médicales, lui répond avec la sévérité d'un janséniste offusqué, comme si le seuil de la bienséance avait été dépassé : «Je ne ferai aucun pas pour l'Art de péter. Vous y soupçonnez quelques bonnes plaisanteries et vous n'y en trouverez aucune. Si vous avez pris ce livre pour un ouvrage de médecine qui put servir aux personnes constipées, vous vous êtes trompé. C'est du papier noirci et rien de plus $»^{17}$. L'Encyclopédie le rangera dans « la bibliothèque scatologique », où figurent en bonne place L'Histoire secrète du Prince Croquéton et de la Princesse Foirette, de Marguerite de Lubet,

(15) RGADA (Moscou), Fonds Stroganov, 1278. Lettre de Gabriel Dubreul à Gilbert Romme, 2 mai 1775.

(16) Idem.

(17) Lettre de Gilbert Romme à Gabriel Dubreul, fin 1775-début 1776, in Gilbert Romme, Correspondance, volume 1, tome 1 (1774-1776), Clermont-Ferrand, Presses universitaires BlaisePascal, 2006, p. 248. 
fille d'un président au parlement de Paris (1745), qui met en scène le roi Pétaut et le ministre Constipati, L'Art de désopiler la rate en prenant chaque feuillet pour se t. le D., entremêlé de quelques bonnes choses, de Joseph Panckoucke (1754), Histoire et aventures de Milord Pet, conte allégorique de Jeanne Fesse, dédié à « Messieurs les vidangeurs de la Ville et Généralité de Paris, seigneurs des basses œuvres du Royaume » (1755), L'Oratio pro crepitu ventris d'Emmanuel Marti (1768), sans compter l'Almanach des chieurs, étrennes odoriférantes dédiées à tous les nez. On réédite en 1790 L'Histoire secrète du Prince Croquétron et de la Princesse Foirette ${ }^{18}$. Le roman et le théâtre érotique, quant à eux, mettent peu fréquemment en scène ce dont on s'amuse encore dans les improvisations scatophiles des foires et des boulevards ${ }^{19}$. On gamahuche, on sodomise, on socratise certes, mais en des espaces d'une luminosité lunaire et d'une pureté virginale qui ne supportent qu'exceptionnellement traces et pestilence (" Des culs ronds sans poils et sans crottes », tels que les réclamait Piron dans ses Doléances au dieu Priape). L'apocryphe Correspondance de Madame Gourdan (1783), hommage à cette grande prêtresse des bordels parisiens, se pare de nombreuses chansons, dont celle de «l'abbé Lapin », célèbre chansonnier du Palais-Royal, sur un certain Robin, homme du peuple et par conséquent susceptible de ces vents prolétaires et bestiaux que la civilité réprouve : "Quand il est en colère, / Il montre son derrière / Et vesse comme un daim $»^{20}$. Dans Le Tempérament, tragi-parade en un acte et en vers de Grandval fils (1756), le prince syrien Ratanphor, se refusant à Fessaride, reine douairière d'Égypte, dont il préfère la fille Bellendraps, lui fait comprendre qu'elle ne vaut pas «le pet d'un âne mort» (scène 7), outrage auquel elle répond en le vouant aux gémonies : "Que quinze fois par mois sa montre se dérange; / Qu'il ne puisse gratter où cela le démange; / Que toujours constipé, jamais un lavement / Ne se puisse glisser jusqu'à son fondement 》 (scène 8). Parue dans les années 1740 et souvent réédité sous couvert d'anonymat, Thérèse philosophe dévoile combien la BoisLaurier sait user des vesses comme d'une bombarde afin d'en finir avec un prétendant importun, un " antiphysique » (c'est-à-dire un amateur de sodomie) :

(18) Roger-Henri Guerrand, Les lieux [...], op. cit., p. 49 et sqtes.

(19) Maurice Lever, Anthologie érotique. Le XVIII ${ }^{e}$ siècle, Paris, Bouquins-Laffont, 2003; Jean-Jacques PAUvert (éd.), Théâtre érotique français au XVIII siècle, Paris, Terrain vague, 1993.

(20) Maurice LeVer, Anthologie [...], op. cit., p. 649. 
« J'étais avertie qu'il devait venir me voir, et quoique je sois naturellement une terrible péteuse, j'eus encore la précaution de me farcir l'estomac d'une forte quantité de navets, afin d'être mieux en état de le recevoir suivant mon projet. C'était un animal que je ne souffrais que par complaisance pour ma mère. Chaque fois qu'il venait au logis, il s'occupait pendant deux heures à examiner mes fesses, à les refermer, à porter le doigt au trou où il eut volontiers tenté de mettre autre chose si je ne m'étais pas expliquée nettement sur l'article. En un mot, je le détestais. Il arrive à neuf heures du soir. M'ayant fait coucher à plat ventre sur le bord d'un lit, puis, après avoir exactement relevé mes jupes et ma chemise, il va, selon sa louable coutume, s'armer d'une bougie dans le dessein de venir examiner l'objet de son culte. C'est où je l'attendais. Il met un genou en terre et, approchant la lumière de son nez, je lui lâche à brûle-pourpoint un vent moelleux que je retenais avec peine depuis deux heures. Le prisonnier, en s'échappant, fit un bruit enragé et éteignit la bougie. Le curieux se jette en arrière en faisant, sans doute, une grimace de tous les diables $»^{21}$.

Mais l'apothéose péteuse la plus éclatante est offerte en 1780 par Caquire, parodie du Zaïre de Voltaire, due à « M. de Vessaire », alias Becombes, ancien conseiller à la cour des monnaies de Lyon, De Comberousse ou de Combles selon les exégètes. Une « dernière édition considérablement emmerdée » de cette tragédie en cinq actes en vers, datant de la Révolution, fait une brève allusion au «petit sans culotte » et à l'assignat (« [Je] viens vous déclarer que de tous vos cacas / Je ne donnerais pas même des assignats », acte IV, scène 2$)^{22}$. Un paratexte fixe les personnages dans leur univers merdoré. Quelques exemples de cette distribution à vesses rebattues, qui n'est pas sans rappeler les humeurs de la Cour :

« Cucumane, en habit turc, avec un turban formé d'un bassin d'étain de chaise percée.

PUPUTANT, en vieil habit de cour bien râpé, couleur musc, un bas roulé, l'autre tombant sur les talons, appuyé sur une béquille.

FoIRINE, en robe appelée chemise, dont le derrière est taché ou moucheté en couleur de merde d'oie.

CAQUiRE, en robe à la sultane, sous laquelle elle a un caleçon en nankin couleur de chair, pour pouvoir fournir décemment les gestes et les atti-

(21) Thérèse philosophe, édition établie par François Moureau, Saint-Étienne, Publications de l'Université de Saint-Étienne, 2000, p. 134-135.

(22) Jean-Jacques PAuvert (éd.), Théâtre érotique français [...], op. cit., p. 429-489. 
tudes convenables à son rôle, ayant une anse de pot de chambre suspendue à son collier.

NÉFLAIRANT, en pantalon, avec une tache noire au milieu du derrière, un fouet à la main, ayant sur le dos un pot de chambre en havresac, rempli de papier gris coupé par morceaux.

Merdillon, en gilet caca-dauphin, sa chemise en partie hors de sa culotte, tachée de chocolat, un cadenas ouvert au soufflet de la culotte, la clef du cadenas en main $[\ldots] »$.

Un tel attelage ne peut que prendre la clé des vents pour rejoindre un très particulier théâtre de la foire. Adieu donc « sexe poli » et « fesses parfumées » : voici venu le temps lourd des coprophages pulsions, des grosses commissions, les échelons liquides ou fermes de l'étron, la prison individuelle de la constipation. "Tu pètes?... Je t'entends », avoue Caquire, amoureuse du sultan Cucumane, ennemi de son père Puputant, à Foirine (scène 1), dans des dialogues sur les fondements qui associent la vue et l'ouie, celle-ci toute entière collée aux pots de chambre. Opposant foireux et consistants, auxquels revient tout le pouvoir, ils tracent les frontières sociales entre l'aristocratique émission, à peine contrariée par les fistules et les hémorroïdes, et « ces bons gros culs bourgeois » et sans manières, qui «de leur liquide engrais fertilisent les terres», non sans assurer quelques promotions, et autant de régressions anales dans la nobilitas :

«Acte I, scène 1.

CAQUIRE : Sois toujours mon égale, et prise mon odeur;

À deux doigts de mon cul sens-en mieux la douceur.

FoIrINE : Hélas! Puisse le ciel souffrir cette équipée!

Puisse cette grandeur, plutôt cette fumée,

$\mathrm{Ne}$ vous porter au nez qu'agréables vapeurs,

Vous sauver des regrets et des remords vengeurs;

N'est-il point en secret de souci qui vous creuse?

Ne vous souvient-il plus que vous fûtes foireuse?

[Arrive Cucumane, N.A.]

[...] On pète vers ces lieux : sans doute, c'est lui-même.

CAQUIRE : Oui, mon cul fait chorus avec celui qu'il aime.

Depuis trois jours, Foirine, absent de ce palais,

Pour me parler de merde il revient tout exprès

Scène 2.

[...] Cucumane : Maître encore incertain d'un vase qui chancelle, 
Je vois ces fiers foireux, traînant leurs culs crottés,

Vouloir aller de pair avec nos constipés;

Et lorsque la trompette étant à mon derrière,

De mon centre à leur nez fait retentir la guerre,

Je n'irai point, en proie à de merdeux amours,

Avec vous les filer aux communs tous les jours.

Acte II, scène 3.

PuPUTANT : À cette cour jadis je fus en temps de foire,

Quand Cupipe à Bovine enchaîna la victoire.

Je combattais alors avec Moncuculi.

Merdun, Foirin, Cunèfle et le fier Chianlit.

Mais, à revoir Paris, je ne dois plus prétendre :

Non... l'âme par le cul je suis trop prêt à rendre.

Je vais, au dieu Foirant, demander aujourd'hui

Le prix de mes coulis tant prodigués pour lui ».

Dubreul n'a évidemment nullement conscience d'entrer dans cet enfer littéraire en demandant L'Art de péter; il participe avant tout à un succès éditorial, propice à maintes rééditions. Pierre Thomas Nicolas Hurtault, maître de pension, ancien professeur à l'École militaire, qui s'intéresse à l'iconographie et à la généalogie des souverains européens, écrira indifféremment un Dictionnaire des homonymes, un Dictionnaire historique de la ville de Paris, des essais médicaux sur les flux menstruels, un poème pour le mariage du Dauphin. Son ouvrage sur les vents paraît dans sa première version en 1751, rue Pet-en-Gueule, au Soufflet (i.e. Paris, chez Jean-Baptiste Langlois), avec cette précision : «À l'usage des personnes constipées, des personnes graves et austères, des dames mélancoliques, et de tous ceux qui sont esclaves du préjugé ». L'édition de 1775 est celle que réclame Dubreul; elle est augmentée de L'Histoire du Pet-en-l'air et de la reine des Amazones, sans compter l'histoire des vidangeurs de Westphalie. Ultime ajout en 1776: «nouvelle édition augmentée de la Société des Francs-Péteurs, pour ceux qui désireraient y être initiés, en Wesphalie, chez Florent Q. », comme il se doit pour un vent qui, partout de même origine, ne connaît pas de frontières. L'épître est adressée « à leurs excellences Messieurs Carnaval et Carême prenant », rattachant donc explicitement le débat sur les flatulences aux réjouissances populaires des calendriers, dont on sait qu'elles ne bannissent pas la scatologie. Décrivant les «attrapes » parisiennes des temps de carnaval, Louis-Sébastien Mercier, dans son Tableau de Paris, dévoile ainsi « mille grossièretés équivoques » qui heurtent désor- 
mais une partie de la bourgeoisie, soucieuse de s'affirmer par une civilité nouvelle, en rupture avec les amusements du commun : un masque en simple chemise arbore sans complexe son recto moutardé, poursuivi par d'autres masques désireux de tremper leurs noirs boudins dans le moutardier; des comédiens du Français jouent des « scaronnades ", n'hésitant pas à vider sur scène un pot de chambre, etc. ${ }^{23}$ Lesage, avant Mercier, avait dessiné les frontières d'un monde intellectuel qui sait rester vent debout. Son héros, Gil Blas de Santillane, va trouver après dîner le poète Nuñez, qui, en brillante compagnie, parle de l'Iphigénie d'Euripide. Tandis que l'un affirme que le danger fait le charme de la pièce, le bachelier Villegas prétend que c'est le vent, celui qui meut les navires de la guerre de Troie et permet de rentrer en Grèce la victoire accomplie ou, s'il leur est contraire, cloue les vainqueurs en Aulide. Son analyse ne cesse de susciter des rires des convives, "qui se mirent à faire à l'envi de mauvaises plaisanteries sur les vents », et le bachelier les traite " d'ignorants et d'esprits vulgaires $»^{24}$.

\section{Les sociétés de francs - péteurs}

Hurtault se fait fort d'apprendre à ses lecteurs les origines, les déclinaisons des flatulences et les meilleures manières d'en disposer (« on peut péter avec règle et avec goût»). Puisant aux sources antiques (Lucien, Hermogène, Quintilien), il glorifie le pet sauveur - retenu, il est réputé corrupteur de l'imagination, provocateur de mélancolie : «Un Pet qui pour sortir a fait un vain effort, / Dans les flancs déchirés reportant sa furie, / Souvent cause la mort / D'un mortel constipé qui touche au sombre bord, / Un pet à temps laché pourroit sauver la vie », traduit plus trivialement : « Pour vivre sain et longuement / Il faut donner à son cû vent ». Hurtault disserte sur la définition même du mot, réfutant Horace qui suggère un tintamarre, considérant de même nature un vent « avec ou sans éclat », tous deux provoqués « par la pituite et les alimens flatueux »; la distinction d'avec le rot tient seulement à la source émettrice. L'auteur, invitant chacun à l'expertise, le nez enfoncé dans l'anus du voisin, distingue entre pets muets, les « vesses » sèches ou foireuses, horriblement

(23) Louis-Sébastien Mercier, Tableau de Paris, op. cit., Paris, rééd. 1990, p. 191.

(24) Lesage, Histoire de Gil Blas de Santillane (1715-1735), Paris, Editions Garnier frères, Classiques Garnier, 1962, tome II, p. 310-312 (la référence se trouve au « livre onzième », chapitre XIV). Merci à Françoise Le Borgne pour cette indication à propos des effets postprandiaux sur les analyses littéraires. 
odoriférantes, et vocaux, les «pétards », subdivisés du vocal plein au semi-vocal : ce dernier, aigu, contraint par l'étroitesse du canal, la faible quantité ou la sécheresse des miasmes accumulés, la mauvaise santé physique du sujet, est soit clair (il est alors dit « pet de demoiselle »), soit moyen, soit aspiré! N'hésitant pas à mettre en branle ses connaissances musicales, et rejetant les cordes pour filer naturellement la comparaison avec les instruments à vent ${ }^{25}$, si appréciés des fêtes populaires et bientôt révolutionnaires, Hurtault classifie aussi entre pets simples et composés, qui offrent successions et gammes harmoniques (récitées en « des syllabes diphtonguées telles que pa pa pax, pa pa pa pax, pa pa pa pa pa pax »), susceptibles de délivrer bien des maisons des esprits malins qui s'y logent ${ }^{26}$. Dénombrant soixante-deux sons, il cite même en exemple un duo d'enfants pétomanes dont il a pu apprécier l'ingénieux concert diatonique, concluant : "Le bas ventre est une espèce d'orgue polyphtongue, où l'on pourroit sans se gêner beaucoup, trouver comme dans un magasin; douze tropes ou modes de sons ». Pour autant, Hurtault, mais sa position de testeur l'y oblige sans doute, conseille en toutes choses le juste milieu, et conclut donc à la bienséance de la seule gamme moyenne. Car le pet, éventuellement associé au rejet d'autres sécrétions (" pisser sans péter, c'est aller à Dieppe sans voir la mer »), devient élément de civilité, de sociabilité, en tout cas de compagnonnage masculin :

«Le Pet affecté [...] ne se passe guères parmi les honnêtes gens, si ce n'est parmi ceux qui logent ensemble, et qui couchent dans le même lit; alors on peut affecter d'en lâcher souvent, soit pour se faire rire, soit pour se faire pièce les uns aux autres, et les faire même si dodus et si distincts qu'il n'y ait personne qui ne les prenne pour des coups de couleuvrines. On peut encore en se couvrant l'anus avec sa chemise, ou pettant et vessant lentement à travers, s'approcher d'une chandelle récemment éteinte

(25) «Une flûte épaisse et large donne un son obscur; une flûte mince et étroite en rend un clair, et une moyenne en rend un mitoyen. Que quelqu'un qui a le vent bon embouche une trompette, il en tirera infailliblement des sons très forts, et le contraire arrivera s'il a l'haleine faible et courte. On tire donc des intrumens à vent, tels que les flûtes, les flageolets, les corps de chasse, etc., des conjectures très certaines sur les différens sons des Pets, c.à.d. qu'on peut par l'expérience de ces mêmes instrumens rendre une raison juste du son perçant ou grave qu'ils rendent lorsqu'on s'en sert».

(26) «Les Pets diphtongues [...] qui s'échappent surtout lorsque le ventre est rempli de raves, d'ails, de pois, de fèves, de navets et d'autres alimens venteux, ingrédiens capables de procurer un son clair, et successif, courts par intervalle, sont des Pets si terribles qu'ils peuvent faire mourir les poulets dans les œufs, tuer le fœtus dans les entrailles de la mère, et faire prendre la fuite au Diable même». 
et essayer de la rallumer, quoiqu'il arrive souvent qu'on ne fasse que la réduire en une poudre ardente qui se dissipe en l'air, ou qu'on se brule le derrière. C'est encore un amusement fort joli que de recevoir une vesse dans sa main, et de l'approcher du nés de celui avec lequel on couche pour lui faire juger du goût ou de l'espèce.

Lautre pet est involontaire; il se fait sans la participation de celui qui lui donne l'être, et arrive ordinairement lorsqu'on est couché sur le dos, ou qu'on se baisse, ou qu'on fait de grands éclats de rire, ou qu'on éprouve de la crainte. Cette sorte de Pet est excusable».

De là une carte de France et une sociologie fantaisistes et grivoises du pet, de celui, réputé rare, de la pucelle, jusqu'aux terribles vacarmes du maître d'armes (« comme ils sont toujours plastronnés, on dit qu'il ne faut les approcher que le fleuret à la main »), en passant par l'indiscrétion de la femme mariée, négligée par le mari et goûtée par l'amant, la flatulence bourgeoise, dodue, accommodée, assortie de son lourd fumet, la perle de bergère aromatisée au serpolet ou à la marjolaine, l'aigre gaz échappé de la vieille femme, etc. Où l'on retrouve les préventions des aéristes vis-à-vis de l'air vicié de la capitale : «Les Pets de province sont pas si falsifiés que ceux de Paris où l'on raffine sur tout. On ne les sert pas avec tant d'étalage; mais ils sont naturels et ont un petit goût salin, semblable à celui des huîtres vertes; ils réveillent agréablement l'apétit »-considérations gourmandes qui font penser au goût de l'olive verte recherché par l'auteur de Juliette dans ses dégustations coprophages.

Si elle est perçue par des observateurs amis comme le triomphe de la raison, la Révolution française ne jette pas de voile pudique sur les jeux du corps. Ils sont crûment évoqués en 1789 dans l'Art de foutre en quarante manières, ou la Science pratique des filles du monde, publié à Amsterdam. Non content de rappeler les cibles favorites des jésuites ( « Pour honorer Loyola / Ils se servaient du trou caca »), l'auteur inconnu s'amuse au jeu de l'escarpolette, si propice aux anatomies peintes plus poétiquement par Fragonard : «Tandis que l'escarpolette / Est bien mise en mouvement, / Je vois le con par-devant, / Et plus bas le trou qui pète $»^{27}$. La Révolution ne fait pas non plus disparaître l'idéal de la Société des francs-péteurs dont se revendiquait Hurtault, « le savant auteur » auquel rend grâce son successeur. Au plus fort de la réaction républicaine de l'an VI en effet, un de ses défenseurs prend à témoin l'opinion publique en publiant un Éloge du Pet. Sous couvert d'anonymat, il donne suffisam-

(27) Patrick Wald-Lasowski, La science pratique de l'amour [...], op. cit., p. 143 et 149. 
ment de pistes sur le reste de son œuvre pour que l'on reconnaisse en lui Claude François Xavier Mercier de Compiègne (1763-1800), polygraphe, éditeur et compilateur bien connu pour ses ouvrages satiriques et libertins, secrétaire du chevalier de Jaucourt jusqu'à la mort de ce dernier, en 1799, secouru par la Convention, puis commis dans les bureaux de la Marine, qui accueillent et protègent nombre d'hommes de plume. Habitué des pages de l'Almanach des Muses, ce libertin publie beaucoup (plus d'une trentaine d'œuvres, datant principalement de la Révolution), nécessités matérielles et carriéristes faisant loi, sans que fortune s'ensuive. En prose ou en vers, il fait sien le domaine de l'érotisme : Les Veillées du couvent, ou le Noviciat d'amour (1793), Nouvelles galantes et tragiques (1793), Origine des puces. Le pucelage conquis : poèmes libres et autres pièces du même genre (1793), Éloge du sein des femmes, Bréviaire des jolies femmes (1798), La Calotine, ou la Tentation de saint Antoine (1800), des traductions (De l'Utilité de la flagellation dans les plaisirs du mariage et dans la médecine et dans les fonctions des lombes et des reins, d'après Johann Heinrich Meibom, en 1795; Le Jardin d'amour, ou le Vendangeur, poème traduit littéralement de l'italien de L. Tansillo, en 1797; Éloges du porc, de la boue et de la paille et autres pièces traduites du latin, en 1798). Des temps politiques, il parle aussi, dans deux œuvres de circonstance (La Fédération, ou Offrande à la liberté française, poème lyrique en un acte, 1790; Les Délassements d'un philosophe, almanach dédié à la Convention nationale, pour faire suite aux Étrennes poétiques et morales, 1795), dans des réflexions esthétiques sur le vêtement national (Comment m'habillerais-je? Réflexions politiques et philosophiques sur l'habillement français, et sur la nécessité d'un costume national, 1793) ou sur les musiques de la Révolution (Les Concerts républicains, ou choix lyrique et sentimental, 1795), dans un témoignage romancé enfin, une réflexion rétrospective, empreinte de spleen et non dénuée d'intérêt, sur la suspicion qui l'a brièvement frappé en l'an II, le conduisant de la Conciergerie à la Maison de Plessis (Les Nuits de la Conciergerie, rêveries mélancoliques et poésies d'un proscrit, fragmens échappés au vandalisme, 1795) - où le citoyen, devant le bûcher des vanités, dialogue avec l'Être Suprême et exalte la solidarité et les vertus de l'amour fidèle; où le littérateur court après les Iambes d'André Chénier...

Son Éloge du Pet ne compte pas moins de 168 pages $^{28}$. Certaines, consacrées aux causes ou à la nomenclature des vents, à leur musicalité, 
sont carrément recopiées de L'Art de péter, cependant actualisées à l'aune des mutations administratives et sociales engendrées par la Révolution, dont on ne sait pas toujours si elle est victime d'une ironie moqueuse de l'auteur, qui en a connu les prisons, ou porteuse de ses espoirs après le 9 Thermidor, placés en l'occurrence bien bas; les deux, sans doute. Ainsi apparaissent en lieu et place des provinciaux les « pets départementaux », ceux des « fournisseurs de la République », ceux des courtisanes du Palais-Égalité, ceux des « petits auteurs », ceux des nouveaux bureaucrates, qui tuent l'ennui ou dédaignent d'interrompre leur tâche " en allant péter dans le corridor », ceux des savants de l'Institut, obligés de " féminiser » leurs flatulences pour « ne pas déranger l'ordre des travaux, des lectures et des motions ", avant de se libérer dans le privé des fèves qu'ils mangent en plus grand nombre que les poulardes (p. 75-77). Jusqu'aux arts de la scène patriotiques qui semblent propices aux moufettes : "Notre théâtre offre tous les jours des innovations si heureuses dans le tragique que je ne serais pas surpris d'entendre une pétarade arrangée par Méhul, Gossec et Chérubini » (p. 79). Les échos de la guerre ne sont pas moins assourdissants dans l'un des poèmes cités, Le pet et le politique, quoique la place publique se limite à une enceinte qu'aurait revendiquée Procope :

«Au café, de grands politiques,

Parlaient entr'eux des affaires publiques;

Tel à la guerre et tel à paix croyait.

Toutefois chacun convenait

Que la guerre serait certaine

Dès le premier coup de canon.

De la triste réflexion

Les pauvres gens très-fort en peine,

Pour mieux penser à cet objet,

Gardaient le plus profond silence.

Un d'eux qui, par ennui, de bien bon cœur dormoit,

Se retourne, s'agite, et lance un ferme pet.

Oh! parbleu, de ce coup je déserte de France,

Dit un milord, qui là pour lors était;

Vous l'avez entendu? L'hostilité commence » (p. 97).

L'Éloge du Pet prétend que sa proscription de la société des hommes est « contraire à la conservation de la République », car attentatoire à la liberté et à la santé individuelle, moins dangereux pour l'ordre public que 
« les filles, les escrocs, les voleurs et les juifs ». Où l'on quitte l'odeur et le ton de la gaudriole pour les noires fumées de la haine sociale et des amalgames xénophobes, histoire de rappeler les limites idéologiques de ces jeux libertins masculins et misogynes, qui défendent le « franc péter contre la faction hypocrite et intolérante des culs serrés ». L'intolérance religieuse est du reste entretenue tout au long du récit, et jusque dans ses notes prétendument savantes et fondamentalement ignorantes : « Les Juifs prétendent que quand ils pètent en faisant leurs prières, c'est un mauvais augure, et un bon lorsqu'ils éternuent. Ils n'osent ni péter, ni allumer leur feu le jour du sabbat. Les Turcs sont de même » (p. 33). À l'élite lettrée, qui y est invitée, est offert un catalogue des exploits de pétomanes - tel ce bossu qui, imitant les sons de la trompette et du clairon, pouvait sonner la charge et la retraite. S'il ne prétend pas rivaliser avec les actions républicaines héroïques consignées plus officiellement par Léonard Bourdon, l'auteur profite cependant de la même mode éditoriale pour la tourner en dérision. Plus encore que les exploits, les historiettes ne cessent d'illustrer le récit : le cardinal du Perron, ponctuant involontairement une partie d'échecs avec Henri IV par le bruit fondamental tout en plaçant un cavalier : "Au moins, sire, ce cavalier n'est pas parti sans trompette »; un favori de Jean IV du Portugal, lâchant un gaz dans la main du roi qui s'appesantissait sur sa fesse et s'excusant d'un mot : « Sire, votre majesté peut-elle frapper jamais à une porte qu'on ne la lui ouvre incontinent? » (p. 103). Mais il s'agit surtout d'un recueil de proverbes, d'un discours sur les origines et d'une anthologie littéraire du pet, qui emprunte essentiellement à la prose antique, n'évite pas les métaphores musicales. Les comédies d'Aristophane (Les Grenouilles, Les Nuées, Plutus) et de Plaute (Curculio) figurent en bonne place, aux côtés des récits de Suétone et de Sénèque - selon les témoignages sollicités desquels l'empereur Claude aurait été vénéré pour avoir « permis de donner l'essor et la liberté aux pets » (p. 35) -, sans omettre La Cité de Dieu de Saint-Augustin! Les Stoïciens sont sollicités. Rutebeuf, Marot et Saint-Évremond ne sont pas ignorés, versifiant pour quelques belles afin qu'elles leur pardonnent leurs échappements solitaires. Une bibliographie rappelle les sources citées et conseille, outre L'Art de péter, présenté comme un pendant de L'Art poétique de Boileau, des traités en latin (Rodolphi Goclenii problemata de crepitu ventris, 1596; Amphitheatrum sapientiae Socraticae joco seriae, publié à Hanovre en 1619 par Gaspard Dornavio) et deux pièces de théâtre : Généalogie de Milord Pet et Caquire.

Mercier de Compiègne se risque à peine à une définition scientifique de l'objet, peu éclairée par les observations de Lavoisier (quoiqu'une dis- 
tillation de la matière éjectée ait été soi disant tentée, jusqu'à en extraire un onguent contre les tâches de rousseur, et qu'il admire beaucoup l'aéromètre inventé par Régnier, du Lycée des Arts) :

« Le pet est un vent renfermé dans le bas ventre, causé par le débordement d'une pituite attiédie, qu'une chaleur faible a atténuée et détachée sans la dissoudre; un air comprimé qui cherchant à s'échapper parcourt les parties internes du corps, et sort enfin avec précipitation quand il trouve une issue que la bienséance empêche de nommer. Sa définition est conforme aux règles les plus saines de la philosophie puisqu'elle renferme le genre, la matière et la différence » (p. 58).

La comparaison est risquée avec le rot, la globalisation avec le cul, « la partie la plus essentielle de l'homme » (« Parler du pet sans parler du cul, c'est décrire Rome sans parler du Capitole », p. 82). De la réinterprétation de la Création - qui conduit à la lourde question de savoir si l'Être suprême souffla, pour l'animer, dans la bouche d'Adam ou dans son antipode -, retenons cette leçon de modelage qui doit peu à Condillac :

« Aussitôt que le suprême architecte de tout ce qui existe eut paîtri de ses divines mains cette superbe bête à deux pieds, sans plumes, appellée Hоммe, lorsqu'il eut soufflé dans le sein de cette masse, encore inanimée et inerte, cet esprit subtil et igné qui lui donna la vie et le mouvement, lorsque cette faculté d'exister eut besoin de se manifester au dehors par l'exercice des fonctions animales, croira-t-on que le modeleur éternel ait ignoré le point le plus essentiel, et qu'il ait omis de donner à sa créature le moyen de pousser au dehors l'air intérieur qui, intercepté dans les capsules, nuisait à la perfection de son ouvrage? Ne voyons-nous pas le figuriste employer tous ses soins pour empêcher les globules d'air de se glisser entre le moule et la cire liquide qui doit se rassasier de l'empreinte? Dieu pouvait-il ignorer ce premier élément de modeler? Non, sans doute. Ensuite, le premier homme, qui ne savait pas encore rougir et ne connaissait pas les lois tyranniques de la civilité, a-t-il pensé, croyez-vous, à comprimer, à étrangler au passage, cet air qui ravageait son sein et cherchait impatiemment une issue? Non, tout s'accorde à nous faire croire qu'il péta au nez de celui qui venait de lui donner l'existence et que l'Être suprême, loin de s'en fâcher, fut si content d'avoir réussi que pour récompenser son ouvrage, il forma le projet de lui donner une compagne. Observez donc, chers frères, que $1^{\circ}$. Nous devons l'origine de la femme à un pet; $2^{\circ}$. Qu'Adam ayant pété avant que de parler, le Pet est incontestablement plus ancien que la parole. Sentez bien mon raisonnement » (p. 6-7). 
L'Éloge du Pet comprend le règlement provisoire de la Société des francs-péteurs, espérée dans ce temps où clubs et cercles ne manquent pas (p. 140-144). Celle-ci s'adresse, c'est bien le moins, à des gens d'aisance, des honnêtes hommes âgés de 24 à 60 ans, cooptés par les deux tiers de leurs semblables après une période probatoire d'un an, durant laquelle les candidats devront faire assaut d'éloquence (discours, poèmes et odes au Pet principalement, la prose étant cependant préférée aux vers, et les madrigaux, quatrains, épîtres, stances et couplets réservés aux jeux de table). Lors de leurs assemblées, les francs-péteurs ornent leur cou d'un ruban blanc au bout duquel pend un Zéphir en or couronné de fleurs, en dessous duquel cette devise : "À la liberté », dans son expression si particulière que rappelle le serment : «Tenant à grand honneur d'entrer dans la société des francs-péteurs, je promets une constante soumission à son directeur et une tendre amitié à tous les frères. Ennemi déclaré du préjugé, je le combattrai en tous lieux, en pétant librement, souvent et méthodiquement ». Toute nouvelle « intronisation » est suivie d'une allègre canonnade de pets de la part des frères, qui usent de la même méthode chaque fois qu'ils veulent applaudir, lors des réunions, occasions de dîners frugaux tous les deux mois dans la «case » qui leur sert de temple. Ces « cases » sont celles de la société mère et des succursales parisiennes (au nombre de trois) et provinciales (une dans chaque ville), qui comptent trente membres au plus et sont toutes contrôlées par une hiérarchie qui emprunte à celle de la franc-maçonnerie pour mieux la contrefaire : un directeur, un vice-gérant, un orateur, un foudroyant, un interlocuteur, un trésorier. Des correspondants sont envisagés dans chaque commune et à l'étranger. Une assemblée générale de toutes les « cases » est convoquée " chaque année le premier ventôse, époque où les vents impétueux sont censés faire le plus de fracas ». La prétention à l'universalité interdit de désigner un chef suprême, un secrétaire général ou perpétuel.

\section{Le bruit du peuple}

Les membres de cet empire de l'étroit milieu, au titre de leur académisme et au nom de leurs académies, subiraient à l'évidence des gerçures s'ils se mêlaient à un concert plus populaire. De ces mondes parallèles et irréconciliables, les dictionnaires portent trace, si l'on peut dire, intercesseurs entre le pouvoir et l'opinion lettrée, outils d'ordre et de reconnaissance sociale. Avec un souci d'entomologiste ou d'ethnologue, leurs auteurs répertorient les expressions du vulgaire, telles que les arts de la scène les rapportent, telles que depuis la rue ou la taverne 
elles sont transmises. Encore que le Dictionnaire du bas langage ou des manies de parler parmi le peuple, publié à Paris en 1808 par d'Hautel et Schoell, fasse un distinguo entre ces deux formes de récolement. Il laisse les farces de cabaret, les impromptus et improvisations des arlequinades boulevardières, les héritages de Sedaine, au Dictionnaire comique, satirique, critique, burlesque de Le Roux (sa première édition date de 1718 et il continue à être réimprimé dans le dernier tiers du XVIII ${ }^{\mathrm{e}}$ siècle), dont il rejette les « inepties » et le but ludique. Lui prétend se réserver les apports anthropologiques ${ }^{29}$, une prétention qui résiste mal à la lecture, tant le plagiat du Le Roux est évident. Le but des classements établis par d'Hautel n'est pas d'édifier un conservatoire du parler poissard mais de contrôler le langage des humbles, d'en interdire l'usage, en dénonçant sa « dangereuse licence »-du moins s'il faut prendre au pied de la lettre un argumentaire introductif qui peut tout aussi bien chercher à se concilier la censure. Fort disert quand il s'agit de décliner « pet », « péter », « pétarade » et « vent », ses diverses définitions offrent un concert d'acceptions morales, signant les défauts de caractère ou de civilité : "Il ne pétera plus. Se dit par ironie d'un homme qui est mort, et pour lequel on n'avoit aucune considération »; "On tireroit plutôt un pet d'un âne mort. Se dit d'un homme avare et dur à la déserre »; "Pète qui a peur. Se dit par plaisanterie aux gens poltrons, pour les défier, les narguer; et pour faire entendre que ceux qui sont peureux ne doivent s'engager dans des affaires périlleuses »- Hébert prétendra que « cette grande sainte de guillotine » fait « vesser de peur» les ennemis de la République ${ }^{30}-$; "Un péteux. Terme injurieux qui équivaut à lâche, poltron, freluquet »; "Péter dans la main. Ne pas tenir sa parole; y manquer dans le moment où la personne à laquelle on l'avoit engagée a le plus besoin de secours »; " Fier comme un pet. Pour dire hautain, orgueilleux; qui affecte l'air méprisant et dédaigneux »; sans compter l'expression si chère au Père Duchesne, demeurée de nos jours, autant que les sujets qu'elle égratigne : "Péter plus haut que

(29) « Le Dictionnaire du Bas-Langage [...] n'ayant aucun but comique on n'a pas cru devoir s'astreindre à y insérer les expressions que l'on trouve dans les auteurs qui se sont adonnés au genre bouffon ou burlesque; outre que ces expressions travaillées péniblement et avec art, n'ont ni l'énergie, ni l'originalité de celles qui sortent sans effort de la bouche du vulgaire, la plupart d'ailleurs ne sont point parvenues jusqu'à lui; on s'est également abstenu d'y faire mention de ces innombrables inepties qu'un des théâtres les plus fréquentés de la capitale voit continuellement naître et mourir presque au même moment; car, on le répète, le but du Dictionnaire du Bas-Langage n'est point de perpétuer ces dangereuses licences, mais, au contraire d'en interdire rigoureusement l'usage, et de conserver à la langue française une célébrité qui lui est si justement acquise ».

(30) Michel Biard, Parlez-vous sans-culotte? [...], op. cit., p. 541. 
son $c u l »$. Le parler cru renvoie plus rarement à une condition sociale : «On dit trivialement, et par raillerie, d'un homme logé au dernier étage d'une maison, qu'Il entend les anges péter ». Il transcende parfois les genres et les classes pour être partagé par tous et s'appliquer avec dérision aux objets à la mode ou au goût ("Pet en l'air. On appeloit ainsi à Paris, il y a quelques années, une espèce de casaquin que portoient les femmes »; « Pet de nonne. Espèce de pâtisserie soufflée »). Il s'applique aux réalités humaines qui subsument volonté et retenue ( Péter comme un roussin. Péter fréquemment »; "Péter à la sourdine. Vesser; lâcher des vents coulis, faire des pets étouffés, qui, sans faire de bruit, se font néanmoins sentir vivement à l'odorat »; "Lâcher un vent, un vent coulis. Pour commettre une incongruité; lâcher un mauvais vent »; «Tomber sur son péteux. Pour se laisser tomber sur le derrière, sur les fesses »). Il se fait enfin métaphore pour mieux susciter le rire : «Un pet à vingt ongles. Manière burlesque de désigner l'enfant dont une fille est accouchée ».

La caricature va s'emparer de cet habitus, fortement encouragée par le pouvoir en l'an II, qui veut déshonorer ses ennemis et vante cette " sorte d'écriture parlée et colorée convenant à merveille aux illettrés », ce peuple enfant qui facilite évidemment le retour au stade anal. Cependant, si des poissards forts en gueule se déculottent à l'occasion pour mieux afficher leur appartenance militante ou leur mépris contre-révolutionnaire, s'ils fessent ou purgent l'adversaire, se servent de ses œuvres comme d'un torche-cul, ils pètent, somme toute, rarement, et font de ce bruit plus ou moins fluet l'arme fatale du discrédit définitif. Ce sont plutôt les redoutables diarrhées d'un garde française, applaudi par un Parisien pour cette solidarité spontanée, qui noient les privilégiés dans Ma finte, pour ce coup$c y, y$ n'en reviendrons jamais (fig. 33), dans un « tête-à-cul» avec les victimes décapitées des événements de juillet 1789, tandis qu'en arrière-plan des aristocrates aiguisent les poignards de leur complot (1790) ${ }^{31}$. Dans L'Égout royal (fig. 34), l'une des charges les plus violentes suscitées par la fuite interrompue à Varennes, c'est le maire de Paris, Bailly, qui défèque sur le dos courbé du roi, rampant dans le cloaque des Tuileries ${ }^{32}$.

Les bruits et les réalités de la guerre surtout, parce qu'ils renvoient à un monde masculin, aux vertus du courage et à leur antithèse, la peur, favorisent toutes les pétarades. Ainsi pour ce régiment prétendument renvoyé de La Rochelle après une dispute avec les gardes nationaux du cru, consé-

(31) Antoine de Baecque, La caricature révolutionnaire, Paris, Presses du CNRS, 1988,

(32) Ibidem, p. 180-181. 
quence du bruit de la discorde, une dragonnade péteuse qui enflamme un cabaret : aux bourgeois de la garde qui se plaignent de l'effluve, l'auteur, «prenant le parti de son derrière auquel il paraît chaudement attaché, répondit que tel était son usage lorsqu'il se trouvait dans une taverne ». Ce que l'observateur de la scène, qui la rapporte à La Rocambole des journaux $^{33}$, s'empresse de remettre à sa juste hauteur : "Sans doute un pet, tant gros qu'il fut, ne mérita jamais qu'on se coupât la gorge, car, à en juger philosophiquement, autant en emporte le vent ». Alors que le conflit n'est pas encore déclaré, les royalistes s'amusent en effet des préparatifs et des provocations aux frontières, comptant sur une défaite française pour rétablir le trône (non sans jouer parfois sur l'ambiguïté du terme), tel le dessinateur de Nouvelle poudre à la Maréchal de la fabrique des sieurs Bender et Cacabeau. Passée dans l'histoire ce 13 février 1792, une aquatinte anonyme (fig. 35). Avec la scatologie qui sied quand on veut renvoyer l'adversaire à son infantilité, sont mis fesse à fesse deux vieillards esquissant un pet de deux : le feld-maréchal baron de Bender, octogénaire commandant de la place du Luxembourg, et le sexagénaire maréchal Jean-Baptiste Donatien de La Rochambeau, héros de la guerre d'Indépendance américaine, qui est en train, côté français, de réorganiser l'Armée du Nord. Le Journal du Peuple, qui publie l'œuvre, commente :

«Le maréchal de Bender et le sieur Cacabeau sont en rase campagne et dans une position telle que, pour le moment, ils ne sauraient la rendre différente. Ils montrent tous les deux ce que Brunel à Marsise montra. Les efforts de Cacabeau qui fait la grimace et qui semble avoir peur, ne produisent que de l'eau claire; mais Bender, souriant malignement, fait partir des boulets dont la direction cause bien de l'inquiétude pour son adversaire $»^{34}$.

À l'heure de la victoire de Valmy, les graveurs n'hésitent pas à adapter des dessins venus d'outre-Manche, telle la Déroute des Prussiens par les sans-culottes qui les renvoient avec Brunsvick en leur faisant cadeau de la foire (fig. 36), dont le thème est emprunté à Isaac Cruikshank, sans ambiguïté sur les manifestations physiques de la débandade. Elles sont commentées au besoin (s'il en est) par le duc défait : "Oh, mes amis, dépêchons-nous, car cette terre de la liberté nous fera mourir de la foire...

(33) $\mathrm{N}^{\circ} 11,5$ février 1792.

(34) Claude Langlois, La caricature contre-révolutionnaire, Paris, Presses du CNRS, 1988 , p. 135 et 219 . 
Je n'en puis plus. Aye, aye » tandis qu'un sectionnaire pousse aux frontières ces « vilains puants » ${ }^{35}$. Même reconduite pour l'émigré représenté en 1792 par un anonyme ( $F i$ donc M. l'émigrant) : il surenchérit dans l'expression («Vous foirez en vannant»), défait par la peur sociale, tandis qu'au ridicule de son plumet un officier de la garde nationale oppose une aristocratie désormais toute républicaine (fig. 37). Il le met littéralement en garde et lui propose un essuie-mains, vénielle erreur d'appréciation sur le quartier des parties nobles à nettoyer, sauf à user de doigts complices ${ }^{36}$. Mais les députés de l'Assemblée nationale savent tout autant retourner leurs liquides intestinaux contre les princes coalisés, qui refluent sous l'averse : «Dans quel état leurs culs mettront nos têtes!» (Bombardement de tous les trônes de l'Europe, et la chute des tyrans pour le bonheur de l'univers, fig. 38) ${ }^{37}$. Le proctologue patriote aurait bien du mal à identifier dans cette cohorte de postérieurs également fendus, que continue de dominer un Louis XVI vomissant, les hérauts les plus tonitruants de l'Assemblée : on les préserve dans l'anonymat, tandis que princes, généraux adverses, et surtout gens de peu, fussent-ils voués corps et âme à la sans-culotterie, n'ont pas droit à ces égards. Il faut bien des espoirs de paix, et tous les jeux de mots sur les bruits qui la précédent, pour que du liquide on passe au volatile et que s'assèchent les humeurs dans l'anonyme Sentence patriotique (fig. 39), entonnée comme il se doit sur l'air Sentir avec ardeur : « Mettez tout préjugé derrière, / Ne vous occupez que de P... aix », implore l'auteur s'adressant à des Législateurs, unis dans un serment qui soulèverait les rideaux de la salle du Jeu de Paume s'il n'était scellé en pleine et bucolique nature, celle-là même avec laquelle semble s'accorder le concert des pétomanes.

Pour le pet sec et sans bavure, l'amateur est bel et bien renvoyé aux tourments de la politique intérieure, aux indigestions provoquées par l'adversaire, au transit qui l'exclut de la scène nationale sans qu'il ne soit jamais dégradé par un bruit qui sortirait de son particulier. Ainsi de la magnifique expulsion des chefs du parti noir de l'Assemblée nationale constituante, l'abbé Maury et d'Éprémesnil, conchiés par deux diables aussi furieux que le Roland de l'Arioste, «à qui chierait le plus puant / sur l'humaine nature ». De leur ultime poussée naît un sombre nuage qui renvoie à leur obscurantisme ceux qui se refusent aux lumières révo-

(35) Antoine de BAECQUE, La caricature révolutionnaire, op. cit., p. 211-213.

(36) Un grand merci à Philippe de Carbonnières et à Pascal Dupuy, qui, sentant bien l'âcreté du sujet, m'ont généreusement ouvert les portes de l'Enfer gazeux du Musée Carnavalet.

(37) Antoine de BaEcQue, La caricature révolutionnaire, op. cit., p. 214-215 et 221. 
lutionnaires (Les deux diables en fureur, fig. 9 cahier couleur). Le diable de Jacques-Louis David, l'une des deux caricatures de commande destinées à fustiger l'ennemi étranger qu'il réalise pour le Comité de salut public en 1793 (Le gouvernement anglais), se pare de tous les emblèmes de la royauté (couronne, sceptre, ordre de la Jarretière), mais souffre d'une déformation du fessier, confondu avec la tête d'un foudroyant et brûlant George III - avant que la folie ne l'accable, il est déjà dénoncé par l'artiste pour la ruine économique à laquelle il voue son pays et ses négociants en entrant dans la guerre. L'Enfer après tout est aussi un lieu commun pour le souffle interdit. Le Diable de Dante ne rassemblait-il pas ses troupes en faisant de son postérieur une trompette : « ed elli avea del cul fatto trombetta »-sentence aujourd'hui revendiquée par des groupes de francs-péteurs que Facebook (mais est-ce la bonne orthographe?) réunit ${ }^{38}$.

Le parti adverse n'est pas mieux traité. Le Dernier effort des Jacobins (fig. 40), calembour facile sur le maire de Paris, Pétion, que seule autorise une représentation animalière outrageante (l'âne présume d'un défaut d'intelligence), en apporte la preuve, commentée dans le Journal de la Cour et de la ville du 15 février $1792^{39}$. La statue en pied de Louis XVI, initialement imaginée pour la place de la Bastille, n'a jamais été édifiée; le renoncement à une figuration équestre devait symboliser la proximité du monarque avec son peuple. La gravure insiste en l'occurrence sur la solidité du roi en majesté, quelle que soit l'étroitesse de sa base, et sur les efforts dérisoires des jacobins de Paris pour le faire chuter. Confronté à des émeutes de subsistances, Pétion s'inquiète à l'époque de voir la Révolution fragilisée par la séparation en gésine entre le peuple et la bourgeoisie, préoccupation qui le rapproche de Robespierre. Les néojacobins du Directoire, dénoncés par Laglade (Je t'atteindrai, courrier, fig. 41), subissent à leur tour les méfaits du pet de l'âne, animal des charivaris qui conduit tout droit un sans-culotte, muni d'un poignard et d'un arrêt de mort en blanc, dans l'antre infernal, la caverne des monstres et des tourments de l'âme, un topique du théâtre politique depuis le 9 Thermidor (pensons à L'Intérieur des comités révolutionnaires de Ducancel, au Brigand d'Hoffman et Kreutzer). Comme sur la scène du reste, aux effets et aux lieux se surimposent des pancartes didactiques («Antre du crime $»)$.

(38) Merci à Gérard Loubinoux pour cette référence à L'Enfer (chant XXI, 139) et à ses déclinaisons sur le prétendu Net.

(39) Claude Langlois, La caricature contre-révolutionnaire, op. cit., p. 129 et 132. 
La librairie Wébert avait, il est vrai, diffusé en février 1792 un Grand convoi funèbre, parade de cirque ou de carnaval, improbable Cour des Miracles qui alignait les derniers ténors du club des Jacobins après la division de juillet 1791 qui avait favorisé la naissance des Feuillants (fig. 42). Danton en ours, Robespierre, Pétion et Roederer en arlequins, Dubois-Crancé en cerbère, Couthon sur ses béquilles, Marat, Condorcet, Fauchet et une députation de la Bouche de Fer (allusion au club du Cercle social), etc., étaient survolés par une Renommée à la trompette mal embouchée, propice au pet dantesque ${ }^{40}$. Cette même allégorie sonore est mise à l'inverse au service de la République (fig. 43) et se fait actrice dans une libre représentation de la panthéonisation de Voltaire, opposant terme à terme d'une part les « vrais citoyens » repoussant le pouvoir tyrannique et sacrifiant au nouvel autel du Panthéon, et d'autre part le culte royal auquel la fuite à Varennes (qui donne le titre de l'œuvre: Journée du 21 juin) a mis un terme, faisant de l'idole un "monstre ». Son buste est renversé par le pied et une flatulence noire, puissante et décisive de la Renommée tandis que de sa bouche elle célèbre Voltaire, élevé au rang des " grands hommes ». N'a-t-il pas inspiré le graveur par ses vers extraits de $L a$ Pucelle:

«La Renommée a toujours deux trompettes

L'une à sa bouche, appliquée à propos,

Va célébrant les exploits des héros,

L'autre est au c..., puisqu'il faut vous le dire.

C'est celle là qui sert à nous instruire

De ce fatras de volumes nouveaux $\rangle^{41}$ ?

Cette Renommée, en tout cas, est une nouvelle fois utilisée en 1793 par le républicain Villeneuve pour discréditer Dumouriez (Le Sauveur de la Belgique, fig. 44), qui vient de trahir et auquel il promet le sort de $\mathrm{La}$ Fayette, alors prisonnier des Autrichiens : «La Renommée, la trompette en pétoire, / Du général annonce la dernière victoire ».

(40) Boyer de Nîmes présente cette caricature dans son Journal du Peuple du 2 février 1792; le Journal de la Cour et de la ville la mentionne dans son $\mathrm{n}^{\circ} \mathrm{du} 4$ février. Sur le premier, cf. Annie Duprat, « Le regard d'un royaliste sur la révolution : Jacques-Marie Boyer de Nîmes », AHRF, $\mathrm{n}^{\mathrm{o}} 337,2004$, p. 21-39. Il existe une version non coloriée de cette gravure, qui présente en fond de scène les ruines de la Bastille.

(41) Cf. Claudette Hould et alii, L'image de la révolution française, Publications du Québec, Musée du Québec, 1989, p. 242. 
Ces brises impétueuses plus ou moins subtiles tendraient à prouver qu'il n'y a pas de fumet sans feu; elles n'annoncent pourtant guère de plus grands dérangements que ces courtes séquences révolutionnaires évanouies dans les rires des spectateurs. Elles portent surtout les miasmes des distinctions sociales et politiques, quoique les émetteurs soient rarement identifiables autrement que sous leurs formes allégoriques, figurées (le noble, l'émigré, le soldat peureux) ou animales, voire au détour d'un calembour. Les voluptés gazeuses des éloges et considérations académiques semblent avoir fait leur temps, réservées au vase-clos d'une oisiveté dénoncée - et que ses bénéficiaires n'osent revendiquer telle qu'une fois le Directoire advenu et l'Institut établi. Confiné dans ses publics, érudits ou populaires, le pet l'est donc aussi dans sa portée. Cependant, plus qu'une virgule, il est interjection, et à ce titre entre dans la grammaire politique pour la posté(rieu)rité. Pour preuve, cette affiche imaginée par Siné (fig. 44 bis, cahier couleur) en faveur de l'éphémère candidature de Coluche à l'élection présidentielle française de $1981^{42}$. Sous couvert de renverser les dernières Bastilles, il transcrit littéralement la douce appellation de sans-culotte, comme le faisaient Outre-Manche en 1792 les artistes de la plume et du pinceau.

Philippe Bourdin Université Blaise-Pascal (Clermont-Ferrand II) Centre d'Histoire « Espaces et Cultures » Maison de la recherche 4 rue Ledru 63057 Clermont-Ferrand CEDEX 1 phbourdin@laposte.net

(42) SINÉ, Soixante ans de dessins, Paris, Hoëbeke, 2009, p. 64. 